\title{
ANÁLISE ESPACIAL DE RISCO E PREVISÃO DE CASOS CONFIRMADOS DO COVID-19 NO ESTA- DO DE GOIÁS
}

\section{SPATIAL ANALYSIS OF RISK AND FORECAST OF CONFIRMED CASES OF COVID-19 IN THE STATE OF GOIÁS}

\author{
Bernard Silva de Oliveira \\ Mestre em Geografia pela Universidade Federal de Goiás \\ Instituto Mauro Borges de Estatística e Estudos Socioeconômicos \\ bernard.oliveira@goias.gov.br \\ Helber de Carvalho \\ Mestre em Geografia pela Universidade Federal de Goiás \\ Instituto Mauro Borges de Estatística e Estudos Socioeconômicos \\ helber.carvalho@goias.gov.br \\ Priscila Midori Miyashita \\ Mestra em Ciências Cartográficas pela Universidade Estadual Paulista \\ Instituto Mauro Borges de Estatística e Estudos Socioeconômicos \\ priscila.miyashita@goias.gov.br \\ Rejane Moreira da Silva \\ Instituto Mauro Borges de Estatística e Estudos Socioeconômicos \\ rejane.silva@goias.gov.br \\ Alessandra Cristina Pereira \\ Graduanda em Engenharia Cartográfica pelo Instituto Federal de Goiás \\ Instiuto Federal de Goiás - IFG Câmpus Goiânia \\ alepereira.amb@gmail.com
} Especialista em Planejamento Urbano e Ambiental pela UniEvangélica Centro Universitário de Anápolis

\begin{abstract}
RESUMO
Objetivo: Analisar o avanço dos casos confirmados da COVID-19 em Goiás nos intervalos temporais de 16 de março à 01 de maio de 2020 e estimar quantidade de casos confirmados nos municípios goianos até o dia 10 maio. Métodos: Cálculo do risco de contaminação nos municípios goianos e do Distrito Federal, elaboração dos mapas de riscos a partir da interpolação destes dados pelo método IDW e análise do padrão de crescimento dos casos por meio dos modelos de regressão (linear e polinomiais) Resultados: Maiores caso da COVID-19 ocorreram nas proximidade dos principais eixos rodoviários: BR-153 e BR-060, intensificação o risco de contaminação nas cidades mais populosas, a capital Goiânia, Aparecida de Goiânia e a região do entorno do Distrito Federal. A regressão polinomial de $2^{a}$ ordem teve o menor erro de previsão que o modelo linear e o de $3^{a}$ ordem. Conclusão: A análise espacial foi precisa na identificação das regiões de maior risco de propagação, com maior intensidade nas principais rodovias federais e nas cidades mais populosas. O modelo de regressão polinomial de 2 a ordem demonstrou eficiente na progressão dos casos confirmados da doença no período avaliado neste trabalho, podendo direcionar ações voltadas à saúde pública.
\end{abstract}

Palavras-chave: Geoprocessamento. COVID-19. SIG. Modelo polinomial.

\section{ABSTRACT}

Objective: Analyze the progress of confirmed cases of COVID-19 in Goiás in the time intervals from March 16 to May 1, 2020 and estimate the number of confirmed cases in municipalities of

Recebido em: 07/08/2020

Aceito para publicação em: 19/11/2020. 
Goiás until May 10. Methods: Calculation of the risk of contamination in the municipalities of Goiás and the Distrito Federal, preparation of risk maps from the interpolation of these data by the IDW method and analysis of the growth pattern cases through regression models (linear and polynomies). Results: The biggest case of COVID-19 occurred in the vicinity of the main highway axes: BR-153 and BR-060, intensifying the risk of contamination in the most populous cities, the capital Goiânia, Aparecida de Goiânia and the region around the Federal District. The 2nd order polynomial regression had the smallest forecasting error than the linear model and the third order. Conclusion: The spatial analysis was accurate in identifying the regions with the greatest risk of spreading, with greater intensity on the main federal highways and in the most populous cities. The 2 nd order polynomial regression model proved efficient in the progression of confirmed cases of the disease in the period evaluated in this study, being able to direct actions aimed at public health

Keywords: Geoprocessing. COVID-19. GIS. Polynomial model.

\section{INTRODUÇÃO}

No início do ano de 2020, a população mundial enfrenta a pandemia do novo coronavírus SARS-CoV-2, conhecido também como COVID-19 (do inglês COronaVIrus Disease, enquanto 19 refere-se ao período em que os primeiros casos foram divulgados). A COVID-19 é uma doença infecciosa causada por um vírus que pode ocasionar ao indivíduo complicações mais severas, como a síndrome respiratória aguda grave. Além dos indivíduos sintomáticos, sua disseminação pode ser efetuada por pessoas assintomáticas, ou seja, sem qualquer sintoma da doença (FAROOQ, 2020). A principal forma de transmissão do vírus ocorre quando as gotículas respiratórias do indivíduo contaminado (normalmente expelidas por tosse, espirro ou durante a fala) entram em contato com pessoas não contaminadas (OPAS, 2020).

O novo coronavírus segundo KOUBAA (2020) é originário da cidade Wuhan/China. Entre o dia 17 de novembro de 2019 e 31 de janeiro de 2020 contaminou cerca de 10.000 pessoas. Dentro dessa estatística, são contabilizados 214 recuperados e 213 mortos. Nesse período, o cálculo da taxa de mortalidade era cerca de $2 \%$. Posteriormente, o vírus disseminou-se pelo mundo através de transmissão externa, ou seja, por viajantes provenientes da China que desembarcavam em outros países.

Em 31 de janeiro de 2020, a Organização Mundial da Saúde (OMS), devido ao surto do novo coronavírus, declarou estado de Emergência de Saúde Pública de Importância Internacional e em 11 de março de 2020 o novo coronavírus foi caracterizado como pandemia. No mundo, até dia 21 de abril, os casos confirmados totalizavam 2.397.216 confirmados, com 162.956 óbitos (OPAS, 2020).

No Brasil, o primeiro caso de COVID-19 confirmado ocorreu no estado de São Paulo no dia 26 de fevereiro de 2020. No dia 03 de março foram contabilizados 488 casos suspeitos (notificados) e dois casos confirmados, sendo eles do sexo masculino (CRODA, 2020). Até o dia 22 de abril o Brasil apresentou 43.079 casos confirmados e 2.741 mortes (OPAS, 2020).

Em Goiás também foi determinada situação de emergência em saúde pública, em razão da disseminação do novo coronavírus, por meio do Decreto $n^{\circ} 9.633$ de 13 de março de 2020, pelo período de 180 dias (GOIÁS, 2020a). O principal intuito do Decreto foi minimizar o efeito da expansão de contaminação do vírus e a possível lotação dos leitos hospitalares. Seguindo essa linha de prevenção da disseminação da doença no estado, o Decreto $n^{\circ} 9.634$ de 13 de março de 2020, suspendeu por 15 dias: eventos públicos e privados de qualquer natureza; a visitação a presídios e centro de detenção para menores; eventos esportivos com portões fechados ao público; aulas escolares, nos estabelecimentos públicos e privados, entre outros (GOIÁS, 2020b). No dia 17 de março, por meio do Decreto $n^{\circ} 9.637$, foram ampliadas as atividades que estariam suspensas temporariamente em Goiás, sendo: feiras livres; cinemas; clubes; academias; shopping; atividades de saúde odontológica, pública e privada; bares; boates; teatros, entre outras. As exceções foram os serviços essenciais, como supermercado e drogarias (GOIÁS, 2020c).

No estado, de acordo com a Secretaria de Estado da Saúde (SES), o primeiro caso confirmado do novo coronavírus ocorreu no município de Goiânia em 02 de março de 2020 (GOIÁS, 2020d). Com a evolução da propagação, no dia 21 de abril de 2020, Goiás possuía 421 casos confirmados. Nesse contexto, den-

DOI: http://dx.doi.org/10.14393/Hygeia16056561 $\quad$ Hygeia $\quad$ v.16 $\quad$ p. 348-361, 2020 página 349


tre as alternativas para melhor superar esses obstáculos, destacam-se as iniciativas que propõem uma compreensão de aspectos geográficos e que proporcionem uma visão integrada do território.

O geoprocessamento tem sido empregado em diversas áreas do conhecimento como uma ferramenta de grande importância nas análises de dados espaciais para tomada de decisão dos gestores públicos. Amplamente difundida na área da saúde pública, o geoprocessamento é utilizado em estudos e monitoramento ambiental, na identificação das áreas de riscos de doenças, analisando o aspecto espaçotemporal (CARDOSO et.al, 2020). Os autores apontam que a análise do espaço e da escala de atuação de um determinado fenômeno é tão importante quanto o fenômeno em si. Neste sentido, considerando a velocidade de disseminação da COVID-19 sob o ponto de vista do tempo e espaço, fica evidente essa afirmação, uma vez que para tomadas de decisão de saúde pública, quantificar somente os números de infectados e vítimas não seria suficiente. Quanto a dinâmica de expansão do número de casos, Penerliew e Petkov (2020), descreve que características demográficas, como a densidade populacional e o número total da população influenciam na disseminação da doença.

Sob esta óptica, o presente estudo tem por objetivos: utilizar ferramentas de geoprocessamento para analisar o avanço dos casos confirmados da COVID-19 no estado de Goiás, nos intervalos temporais de 16 de março à 01 de maio de 2020; e estimar (projeção) de casos confirmados da COVID-19 nos municípios goianos, por meio de modelo regressão polinomial para o período de 03 de maio à 10 de maio de 2020.

\section{MATERIAIS E MÉTODOS}

\section{Área de estudo}

A área de estudo selecionada foi o estado de Goiás, localizado na região Centro-Oeste do Brasil, situando-se entre as latitudes: $12^{\circ} 23^{\prime} 44^{\prime \prime}$ e $19^{\circ} 28^{\prime} 58^{\prime \prime}$ Sul e longitudes $53^{\circ} 14^{\prime} 52^{\prime \prime}$ e $45^{\circ} 54^{\prime} 23^{\prime \prime}$ Oeste. Goiás é composto por 246 municípios e seu território é de 340.106 km², conforme a Figura 1 (IMB, 2018).

Figura 1 - Mapa de localização da área de estudo

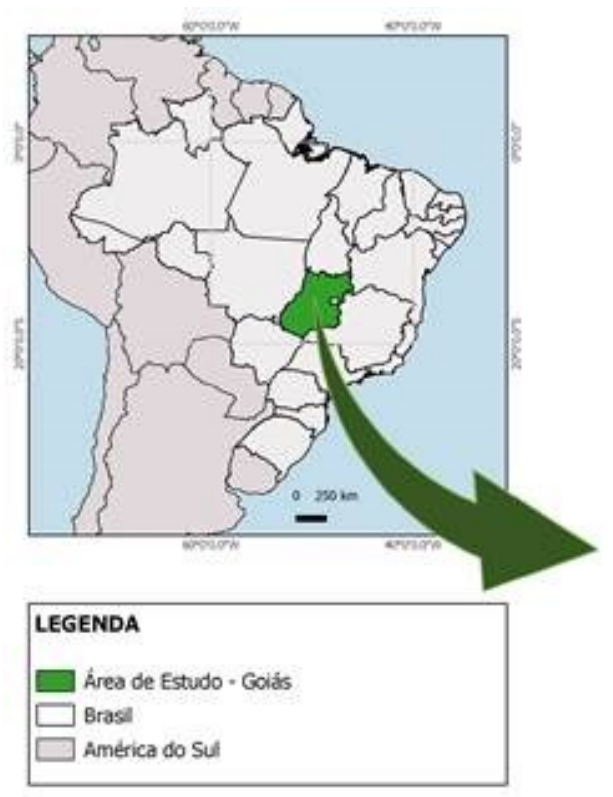

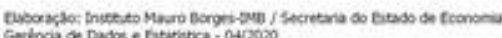

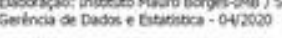

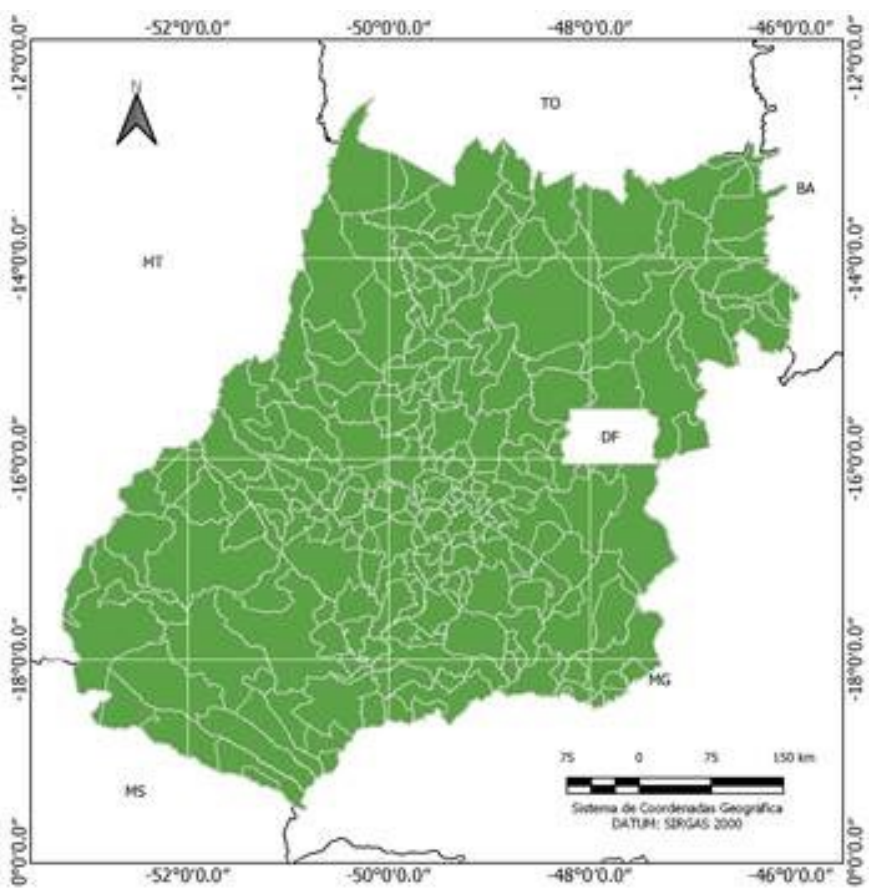


O clima predominante no estado é o Tropical, com presença de duas estações bem definidas: um verão úmido e um inverno seco, com temperaturas médias variando entre $18^{\circ}$ e $26^{\circ} \mathrm{C}$. $\mathrm{O}$ índice pluviométrico acontece predominantemente entre os meses de setembro a abril, oscilando entre 1.200 a $2.500 \mathrm{~mm}$, com a ocorrência de chuvas mais concentradas no verão (IMB, 2018).

\section{Base de dados}

As bases de dados utilizadas neste trabalho foram:

- Dados oficiais da Secretaria de Estado da Saúde de Goiás sobre os casos confirmados da COVID-19 nos municípios goianos;

- Dados oficiais da Secretaria de Saúde do Distrito Federal (DF) sobre os casos confirmados da COVID-19;

- Dados de Projeção da População para todos os 246 municípios do estado de Goiás referente ao ano de 2020, disponível no Banco de Dados Estatísticos do Estado de Goiás (BDE-GOIÁS, 2020);

- No caso do Distrito Federal, utilizou-se o valor da população estimada de 2019, obtida no site do Instituto Brasileiro de Geografia e Estatística (IBGE, 2019);

- Malha oficial dos limites municipais de Goiás e do Distrito Federal georreferenciadas, no formato Shapefile da Environmental Systems Research Institute (ESRI), disponibilizada pelo Instituto Mauro Borges de Estatísticas e Estudos Socioeconômicos (IMB, 2017).

\section{Procedimento metodológico}

Para avaliar o avanço do risco de contaminação da COVID-19 nos municípios goianos, considerou-se também os dados relativos ao Distrito Federal e seu entorno, uma vez que a forte influência dos casos da doença nessa região pode ser causada pela elevada taxa de migração pendular e a sua localização geográfica. Essas condições podem favorecer a disseminação do vírus e influenciar o risco de contaminação, sobretudo, nos municípios goianos por causa da proximidade e da forte dependência de suas populações aos bens, serviços e equipamentos públicos e privados disponíveis no Distrito Federal.

Para a elaboração do modelo matemático de risco de contaminação para cada um dos municípios goianos, utilizou-se como referência a equação da taxa de mortalidade descrita por Oliveira et. al (2013), que considera a razão entre o número de casos de mortalidade registrado pelo tamanho da população em risco. No entanto, para acompanhar a evolução do risco de contaminação de Covid-19, em um determinado intervalo de tempo, foi necessário acrescentar um parâmetro para relacionar a variação do número de casos confirmados em um dado período, resultando na formulação da seguinte Equação (01).

$$
\begin{aligned}
& \text { Risco }=\frac{\text { número de contaminados do município }}{\text { População do muncípio }} * 100.000_{\text {hab }} * K_{\text {mun }} \\
& K_{\text {mun }}=\frac{\Delta_{N C}}{N C_{\text {total }}}
\end{aligned}
$$

Onde:

$$
K_{\text {mun }}={ }^{\top} \text { Variação relativa entre os casos confirmados da COVID-19 em um determinado período; }
$$




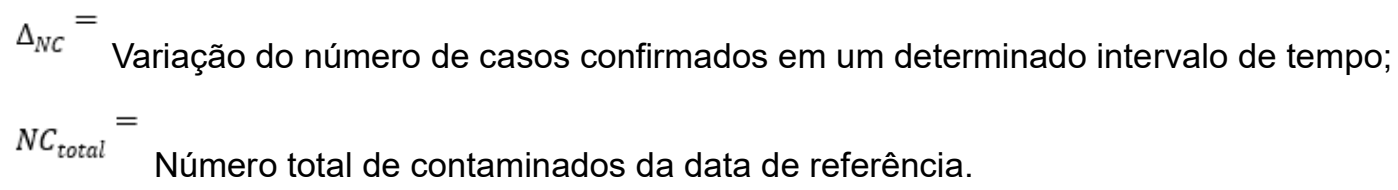

Após calcular os valores do risco para os 246 municípios utilizando a Equação (01), foram elaborados os mapas de riscos, e para isso foram gerados os centroides dos 246 polígonos das divisões municipais do estado de Goiás e um do DF. Utilizando como dado de entrada um shapefile de geometria pontual representando as 247 feições (municípios goianos e DF) juntamente com seus respectivos valores de risco calculados, aplicou-se o método de interpolação pela Ponderação do Inverso da Distância (Inverse Distance Weighting - IDW), utilizando o software ArcMap (ArcGIS). O interpolador IDW adota o modelo estatístico denominado "Inverso das Distâncias", baseado na dependência espacial. Este método supõe que quanto mais próximo os pontos estiverem um do outro, maior a correlação entre seus valores. Dessa forma, atribui-se maiores pesos para as amostras mais próximas, do que para as amostras mais distantes do ponto a ser interpolado (VARELLA; JUNIOR, 2008).

Para elaboração dos mapas de risco a fim de analisar a evolução espacial dos casos da COVID-19, foram definidos três períodos: (I) 18 de março até 01 de abril, (II) 01 de abril até 15 de abril e (III) 15 de abril até 01 de maio. Na etapa de elaboração dos mapas de risco, a definição das classes foi realizada seguindo a distribuição quantil, sendo que para cada período, os valores de risco calculados foram ordenados e divididos iguais, sendo distribuídos em cinco classes: Baixo, Médio-Baixo, Médio, Médio-Alto e Alto. Exceto, no caso dos municípios que tiveram como valor de risco igual a zero, pois esses foram adicionados diretamente na classe de baixo risco.

Findada a elaboração dos mapas de risco, iniciou-se a análise do comportamento de expansão dos casos da COVID-19 em Goiás. Para a identificação dos modelos mais ajustados ao crescimento dos casos no estado foi utilizado a linguagem Python e a biblioteca Scipy para comparar os modelos de regressão simples lineares e polinomiais, por ser uma análise univariada (números de casos confirmados). Com a definição do modelo com maior representatividade, os valores dos possíveis riscos de casos da COVID19 foram estimados.

Com os valores estimados calculados, utilizou-se o mesmo método de interpolação aplicado para visualizar os focos de futuros casos, modelados pelo modelo com melhor representatividade em Goiás. O cálculo da predição baseia-se nos municípios goianos que tiveram casos confirmados no período de um mês. Para aprimorar a estimativa da projeção, o modelo considerou o primeiro dia com caso confirmado em cada município. No dia 16 de março, apenas Goiânia, Anápolis e Rio Verde possuíam casos, portanto para efeito de cálculo, o período estabelecido para predição foi entre 16 de março e 16 de abril. No decorrer dos dias com a disseminação da doença no território goiano, outros municípios apresentaram casos, atingindo em 16 de abril, 42 municípios.

Para validar o modelo foi estabelecido o dia 16 de abril como dia base e efetuada uma projeção para os próximos 10 dias (até 26 de abril de 2020), com cálculos de coeficientes para todos os 42 municípios com casos confirmados, até o dia base, com o intuito de avaliar a relação entre os valores projetados e os respectivos valores de casos confirmados.

Por fim, com o modelo de regressão adquirido, estimou os valores de novos casos para os dias 03 à 10 de maio, uma vez que havia dados divulgados pela SES-GO de casos confirmados só até 02 de maio. Estes valores estimados foram espacializados para verificar a expansão de contaminação da COVID-19 no estado de Goiás.

\section{RESULTADOS E DISCUSSÕES}

Os primeiros casos da doença causada pelo coronavírus (Sars-Cov2), responsável pela COVID-19, foram confirmados em Goiás a partir dia 02 de março de 2020, entretanto somente no dia 12 de março os da- 
dos acumulados diários começaram a ser divulgados pela SES-GO. Em 18 de março, Goiás já havia confirmados 12 casos, sendo cinco em Goiânia, três em Rio Verde, dois em Anápolis, um em Aparecida de Goiânia, um em Jataí e 230 casos suspeitos estavam em investigação. Até essa data ainda não havia confirmação de óbitos no Estado. No dia 01 de maio, Goiás já registrava 808 casos positivos para a doença e um total de 9.636 casos suspeitos em investigação. Nesse mesmo período já haviam 29 óbitos confirmados e quatro suspeitos.

Como a doença estava em evolução e pouco se sabia sobre o espraiamento do contágio utilizou-se os períodos: (I) 18 de março à 01 de abril, (II) 01 de abril à 15 de abril e (III) 15 de abril à 01 de maio para elaboração de uma metodologia a fim de obter risco de contágios para cada um dos 246 municípios goianos.

A Tabela 1 apresenta a análise descritiva dos dados tabulares nos períodos em que foram calculados o risco de contágio. Dentre os municípios goianos, a capital Goiânia teve a maior variação, ou seja, aumento de casos confirmados da COVID-19 com valores máximo de 37 (período I), 134 (período II), 281 (período III) e 457 (considerando todo os períodos). O número de municípios com casos teve uma taxa de crescimento de $7 \%$ no primeiro período, $15 \%$ no segundo período, $18 \%$ no terceiro período, e $24 \%$ considerando desde o início do primeiro caso, reforçando a tendência crescente de casos no estado.

Tabela 1 - Estatísticas descritivas dos dados tabulares de casos confirmados da COVID-19 nos períodos estipulados.

\begin{tabular}{lccc}
\hline \multicolumn{1}{c}{ Período } & Máximo & $\begin{array}{c}\text { Média } \\
\text { (Municípios com casos) }\end{array}$ & $\begin{array}{c}\text { Média } \\
\text { (Municípios sem casos) }\end{array}$ \\
\hline 18 de março à 01 de abril (I) & 37 & 3,75 & 230 \\
01 de abril à 15 de abril (II) & 134 & 6,27 & 209 \\
15 de abril à 01 de maio (III) & 281 & 11,45 & 202 \\
18 de março à 01 de maio & 457 & 13,69 & 187 \\
\hline
\end{tabular}

Fonte: Secretaria de Estado da Saúde (SES-GO), 2020. Elaboração: Autores

Ao analisar a concentração de casos da COVID-19 de forma isolada nos diferentes municípios, verificouse que não seria possível uma análise da interpretação da sua distribuição e visualização no espaço que compreendesse integralmente o território goiano. Desta forma, considerando a necessidade de analisar a evolução do fenômeno no território goiano por completo usou-se a interpolação para transformar os dados pontuais em uma superfície contínua. O mapa de calor é bastante utilizado na análise espacial por permitir a visualização dos locais com maior densidade de determinado fenômeno pontual a partir da avaliação de sua distribuição no espaço. Esse método usa a vizinhança em torno de cada ponto, ou seja, a interpolação como ferramenta para obter informações em locais de forma indireta.

\section{Mapas de risco do COVID-19 em Goiás}

A distribuição espacial do mapa de risco demonstrou inicialmente os municípios (regiões) com os maiores riscos de contaminação por COVID-19, e que necessitavam de maior atenção por parte da população, que são:

- Os municípios do entorno do Distrito Federal;

- A capital Goiânia, estendendo pela região metropolitana;

- A região vizinha ao município de Rio verde;

- As regiões próximas a Paranaiguara e Itumbiara. Embora avaliando a dimensão espacial, com uma área de menor abrangência, o risco foi categorizado como alto; 
- O eixo Goiânia-Brasília.

A Figura 2 mostra a distribuição do risco de contaminação da COVID-19 nos municípios do estado de Goiás entre 18 de março e 01 de abril de 2020 (período l).

Figura 2 - Mapa de Risco de contaminação por COVID-19 (Período I).

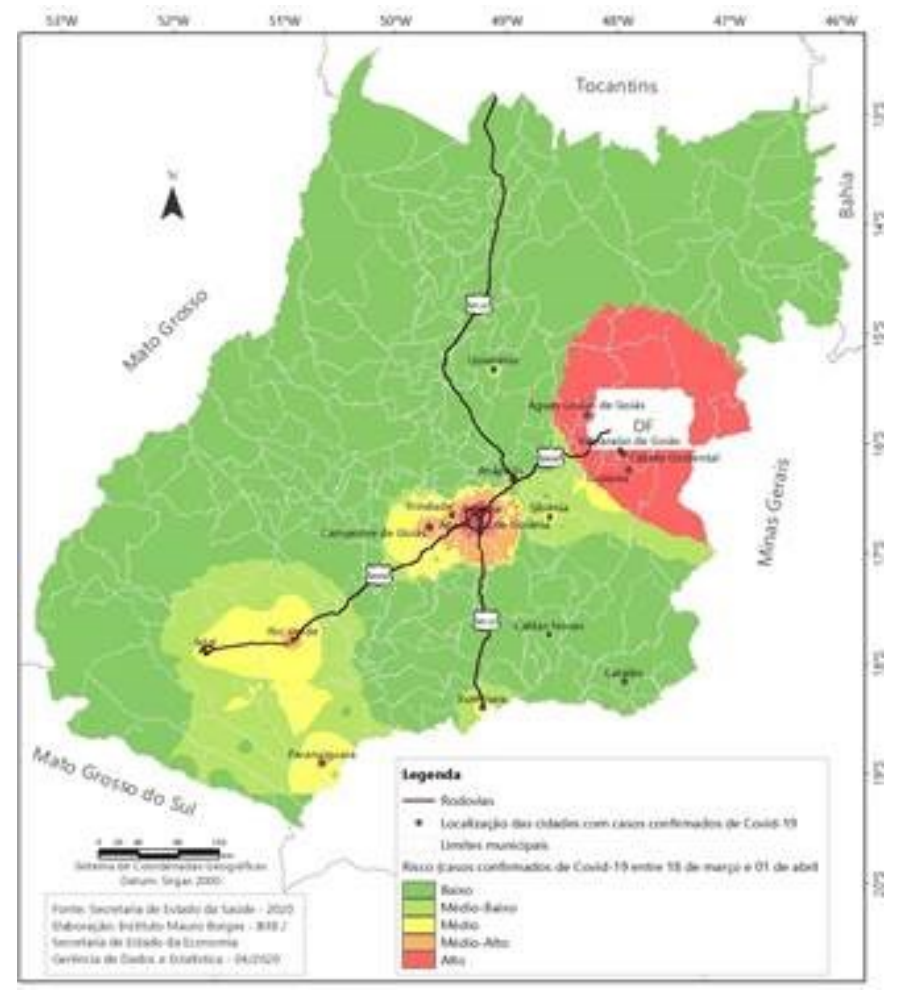

A Figura 3 mostra a distribuição do risco sobre os municípios do estado de Goiás entre 01 de abril e 15 de abril (período II), já com a vigência do Decreto $n^{\circ} 9.633$ e do Decreto $n^{\circ} 9.634$, ambos do dia 13 de março de 2020, e do Decreto $n^{\circ} 9.637$ do dia 17 de março de 2020, que ditam sobre o fechamento das atividades durante a quarentena em Goiás.

Na Figura 3, verifica-se que na segunda quinzena após os primeiros casos confirmados da COVID-19, observa mudança na "dinâmica geográfica" do vírus no território goiano, ou seja, houve um espalhamento dos casos, consequentemente elevando o risco de contágio em novos municípios. Dentre as principais alterações pode-se destacar:

- De forma geral, nota-se um agravamento nas regiões próximas aos principais eixos rodoviários, dentre eles a BR-153 e BR-060;

- Intensificação do risco de contaminação na capital Goiana e nos municípios ao redor;

- O município de Goianésia intensificou o risco de contaminação, tornando-se uma área com potencial de risco aos seus municípios vizinhos;

- Os municípios circunvizinhos à região do entorno do Distrito Federal mantiveram um alto risco de contaminação. Essa manutenção ocorreu possivelmente em virtude da grande influência dos casos do DF nos municípios goianos do entorno. O Distrito Federal apresentou aumento significativo nesse período; 
- Foco em Paranaiguara não se repetiu, o que pode ser explicado pelas medidas de isolamento social que foram instituídas pelos decretos estaduais;

- A região de Rio Verde apresentou uma diminuição na área de influência, ou seja, uma redução do risco ao seu entorno. Possivelmente, essa diminuição pode ter ocorrido influenciada por decretos municipal, que declara situação de emergência em saúde pública e dispõe sobre medidas preventivas de enfrentamento da pandemia provocada pelo Coronavírus, bem como por informações noticiadas pelos veículos de comunicação local, alertando a população sobre o aumento da doença.

Figura 3 - Mapa de Risco de contaminação por COVID-19 (Período II).

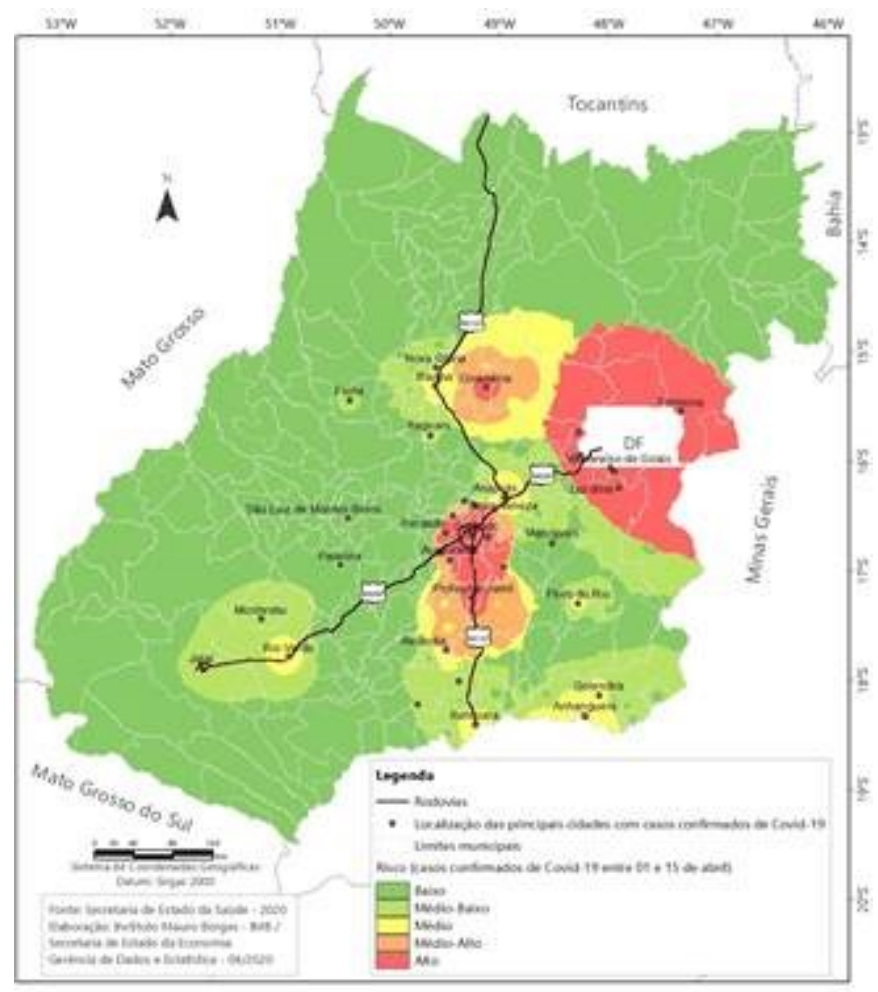

A Figura 4 refere-se ao período entre 15 de abril e 01 de maio (período III), e a "dinâmica geográfica" acompanha a tendência da quinzena anterior (Figura 3). Verifica-se que os maiores riscos de contaminação pelo vírus ocorreram na capital do estado e em seus arredores e na região do entorno do Distrito Federal. É notável a redução do risco de contaminação no município de Rio Verde, indicando que as medidas preventivas, como o isolamento social implementados podem ter surtido efeito na região.

Após a confrontação temporal e análise dos resultados, verifica-se que o mapa de risco da COVID-19 permite acompanhar periodicamente a evolução espacial da taxa de contágio nos municípios, além de favorecer a avaliação das medidas de gestão pública, tais como os decretos regulamentadores. Isso proporciona visualizar quais regiões que possivelmente tiveram ações mais efetivas no controle da disseminação da doença, além da deteç̧ão das áreas que necessitam de maior atenção do sistema de saúde. Dentre os aspectos que se destacaram, está o agravamento dos casos nas proximidades dos principais eixos rodoviários, sobretudo nas Rodovias BR-153 e BR-060, além da intensificação do risco de contaminação nas cidades com maior população, como exemplo deste padrão: a capital Goiânia e o município de Aparecida de Goiânia, além da região do entorno do Distrito Federal. 
Figura 4 - Mapa de Risco de contaminação por COVID-19 (Período III).

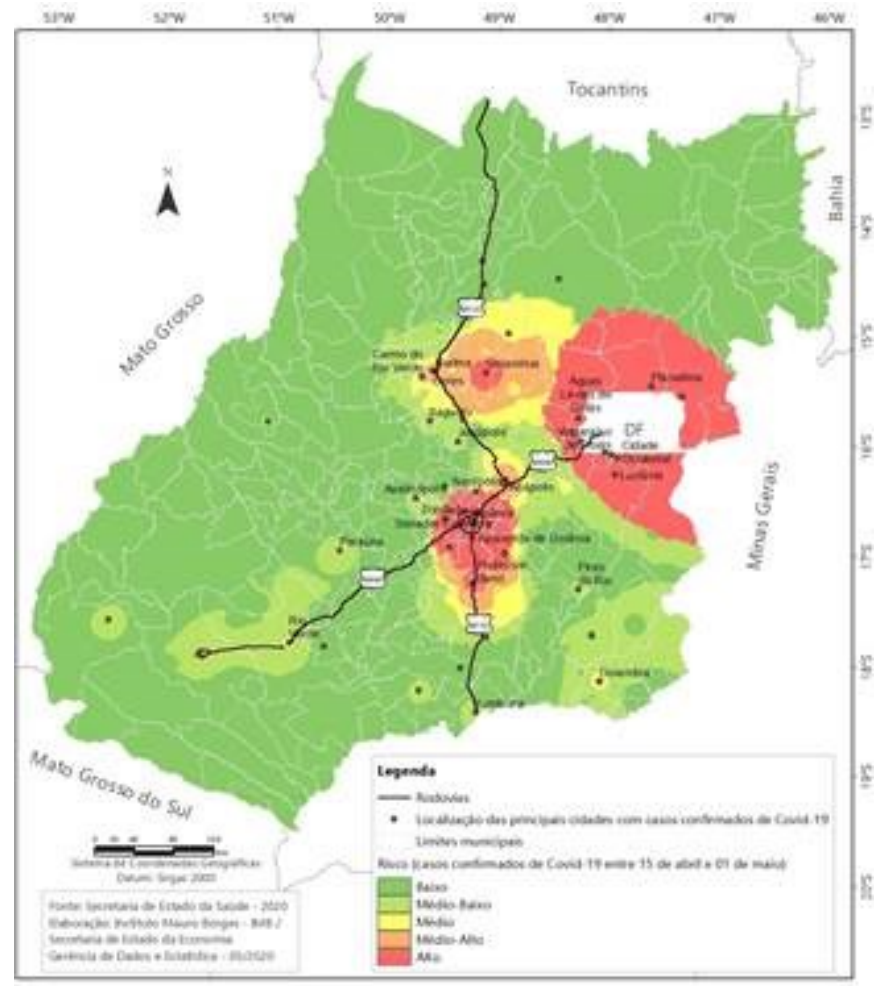

Análise dos modelos de avanço da COVID-19 e distribuição geográfica dos valores preditados em Goiás

A Figura 5 exibe modelo de regressão linear e a Figura 6 o modelo polinomial testado para a identificação do modelo com maior assertividade em relação a casos confirmados da COVID-19 em Goiás

Figura 5 - Modelo linear aplicado em todos os casos confirmados da COVID-19 em Goiás.

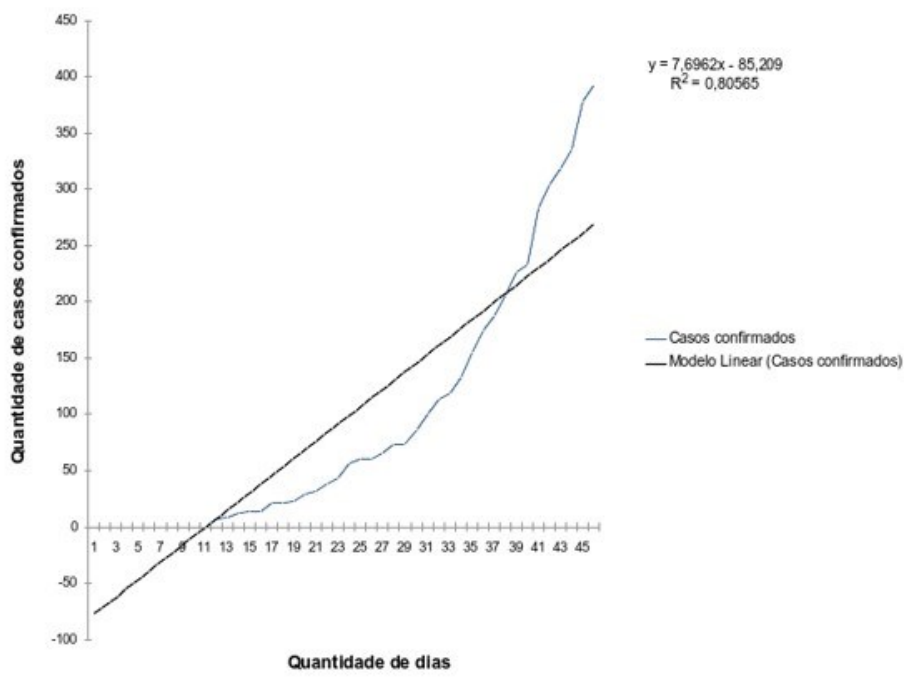


Figura 6 - Modelo polinomial: (a) $2^{\mathrm{a}}$ Ordem e (b) $3^{\mathrm{a}}$ ordem aplicado em todos os casos confirmados da COVID-19 em Goiás.

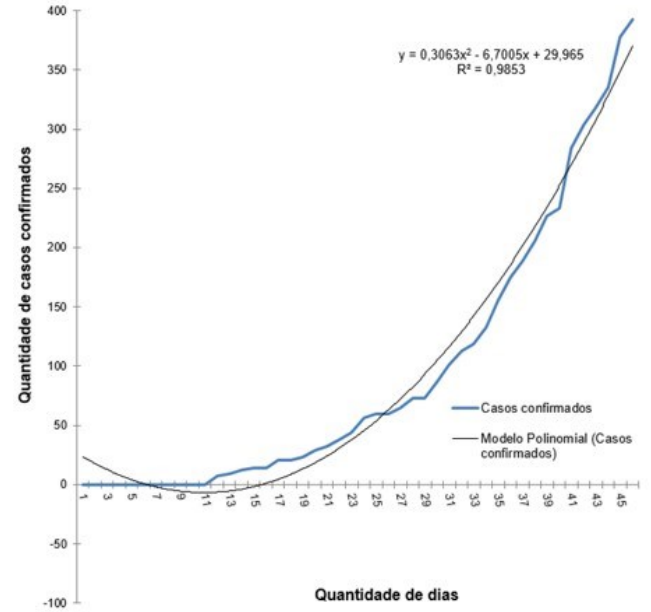

(a)

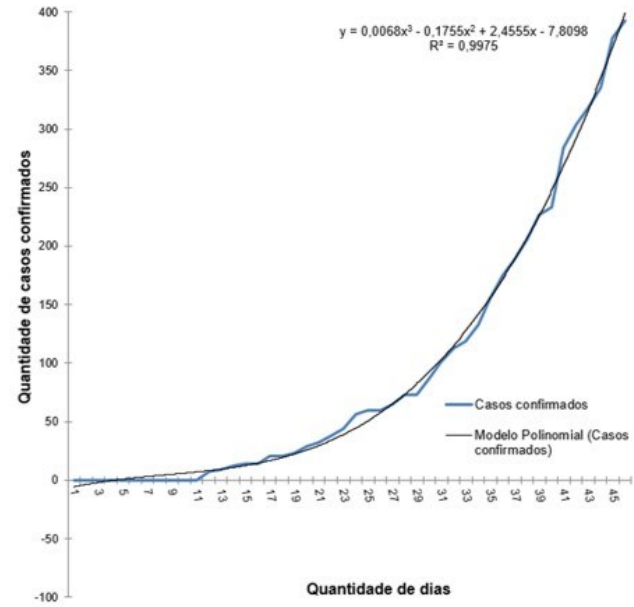

(b)

O coeficiente de determinação que mensura o ajuste da curva obtido pela regressão linear simples foi de 0,80 , isso significa um bom ajuste. Entretanto, devido a tendência de crescimento dos casos e a temporalidade das informações (curta), com uma maior temporalidade dos dados ocorrerá um incremento do erro de previsão ocasionando, em uma série temporal mais longa, em underfitting, isto é, o modelo seria incapaz de estimar os valores de casos confirmados em Goiás em um maior período de tempo.

Como o caso do polinômio foi mais adequado para representar a curva de contaminação da COVID-19, dentro deste modelo foi selecionado o de segunda ordem em vez de ordens superiores, pois os modelos de ordem superior ocasionaram um modelo com problema de overfitting, que significa que o modelo foi capaz de "decorar" os dados para estimar ocasionado erros na hora de prever dados desconhecidos.

A Figura 7 mostra os erros estimados dos casos confirmados da COVID-19 entre os polinômios de $2{ }^{a}$ e 3 a ordem.

Figura 7 - (a) Dispersão do erro do polinômio de $2^{\mathrm{a}}$ ordem; (b) Dispersão dos erros do polinômio $3^{\mathrm{a}}$ ordem.

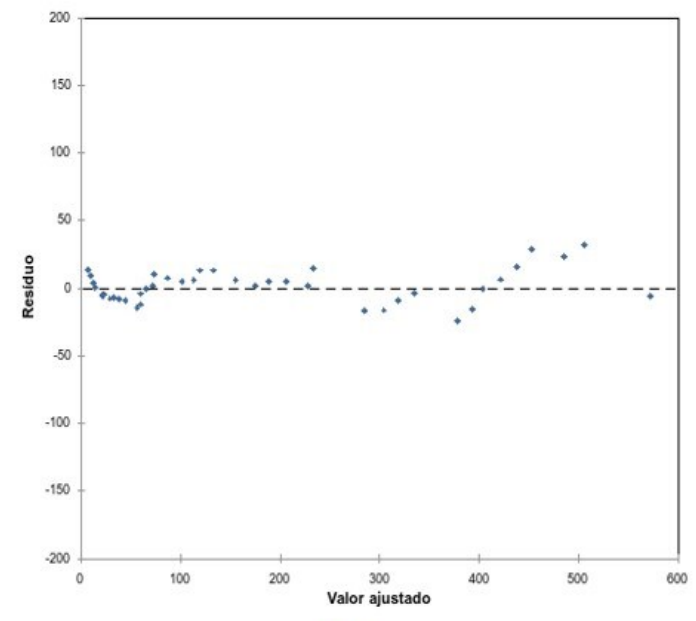

(a)

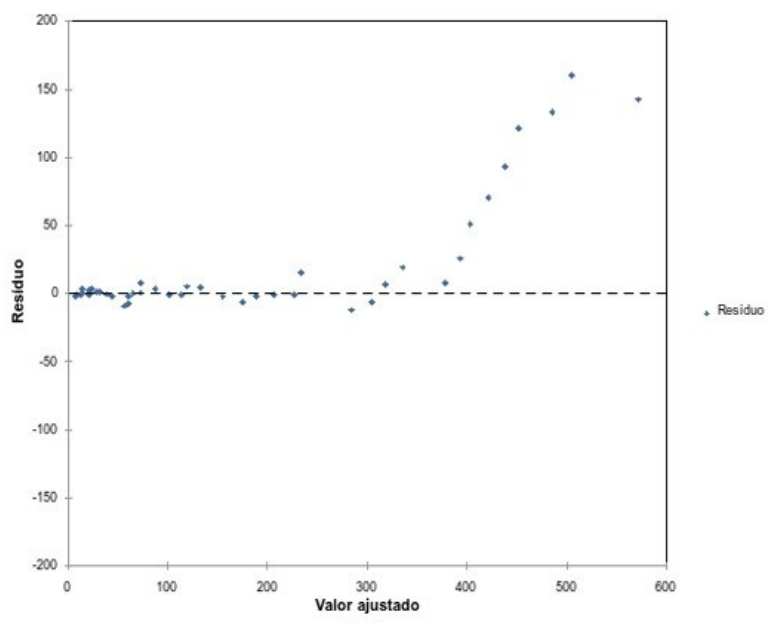

(b) 
Conforme demostra a Figura 7, o polinômio de $2^{\mathrm{a}}$ ordem obteve melhor ajustamento e erros estimados nas predições com maior similaridade entre os casos confirmados com as previsões. Isso ocorre, pois esse polinômio é menos "fino" no ajuste que o de $3^{\mathrm{a}}$ ordem, fazendo com que os erros de previsões ao longo do tempo sejam generalizados entres os dias, como podemos verificar na Figura 6. Das projeções, apenas um município apresentou total inadequação na predicação, com correlação negativa. Essa circunstância é ocasionada porque o município em questão não teve avanço de casos confirmados da COVID-19, mas o modelo estimou o valor por meio de seus pesos já atribuídos.

A Tabela 2 demonstra a diferença (em valores absolutos) entre os casos projetados e confirmados para os municípios avaliados com a adoção da função quadrática. Apresenta o somatório de casos confirmados e projetados nos 42 municípios goianos que tiveram casos registrados no período entre 16 de março e 16 de abril de 2020. Dessa forma, foi disponibilizada uma série histórica mínima, fornecendo insumos para o cálculo da projeção de casos, utilizando para a predição dos dados o modelo polinomial de $2^{a}$ ordem.

Tabela 2 - Comparativo dos valores de casos confirmados reais e projetados do COVID-19 em Goiás

\begin{tabular}{cccc}
\hline Dias & Projetado & $\begin{array}{c}\text { Casos } \\
\text { Confirmados }\end{array}$ & $\begin{array}{c}\text { Diferença } \\
\text { (Absoluta) }\end{array}$ \\
\hline $17 / 04 / 2020$ & 335 & 333 & 2 \\
$18 / 04 / 2020$ & 356 & 375 & 19 \\
$19 / 04 / 2020$ & 377 & 389 & 12 \\
$20 / 04 / 2020$ & 398 & 398 & 0 \\
$21 / 04 / 2020$ & 419 & 415 & 4 \\
$22 / 04 / 2020$ & 440 & 432 & 8 \\
$23 / 04 / 2020$ & 462 & 444 & 18 \\
$24 / 04 / 2020$ & 483 & 469 & 14 \\
$25 / 04 / 2020$ & 505 & 485 & 20 \\
$26 / 04 / 2020$ & 526 & 550 & 24 \\
\hline
\end{tabular}

Fonte: Autores

Analisando a Tabela 2, a maior discrepância da estimativa de casos confirmados da COVID-19 no período avaliado, ocorreu em 26 de abril de $2020 \mathrm{com}$ a diferença de 24 entre casos confirmados e projetados. Entre as diferenças, de casos confirmados e projetados, observa-se que parcela significativa ocorreu na capital Goiânia com 15 casos. Outro aspecto importante, que a diferença no dia 26/04/2020, mesmo constituindo a mais relevante, não acarretou problemas de confiabilidade do modelo, pois esta diferença foi generalizada em relação a quantidade de total de casos confirmados reais da COVID-19, não superestimando muitos os valores e também não gerando falta de confiança ao modelo. A predição dos casos confirmados da COVID-19 para os dias 03 à 10 de maio, a partir do polinômio de $2^{\mathrm{a}}$ ordem é demostrado na Figura 8.

Conforme a Figura 8, o padrão de contaminação está concentrado nas regiões do entorno do Distrito Federal. Isso é ocasionado pela influência dos casos confirmados no Distrito Federal para o entorno devido à migração pendular, uma vez que esses municípios pertencem a Região Integrada de Desenvolvimento do Distrito Federal e Entorno (RIDE), no qual serviços relacionados em especial à áreas de infraestrutura e emprego, são comuns ao Distrito Federal e aos municípios que a integram. Outra região de concentração foi a região Centro-norte Goiano, caracterizada por municípios com maiores populações do estado como por exemplo Goiânia (capital do estado) e Aparecida de Goiânia, com a população estimada em 2019 de 1.516.113 e 578.179, respectivamente. Além disso, nessa região é localizada uma das principais infraestruturas logísticas do estado, rodovias BR-153 e a BR-060, utilizadas para escoamento da 
Análise espacial de risco e previsão de casos confirmados do

produção e deslocamentos intraestadual e interestadual de pessoas, concentrando diversos equipamentos urbanos públicos e privados, como indústrias, comércios e setor de serviços. Esses centros urbanos, em virtude de sua hierarquia, infraestrutura e importância, constituem polos de atração que promovem intenso fluxo, com forte movimentação da população de municípios vizinhos para obtenção, por exemplo de serviços, de saúde ou serviços específicos disponibilizados nesta região.

Figura 8 - Espacialização da predição dos casos confirmados da COVID-19 a partir do polinômio de $2^{\mathrm{a}}$ ordem.
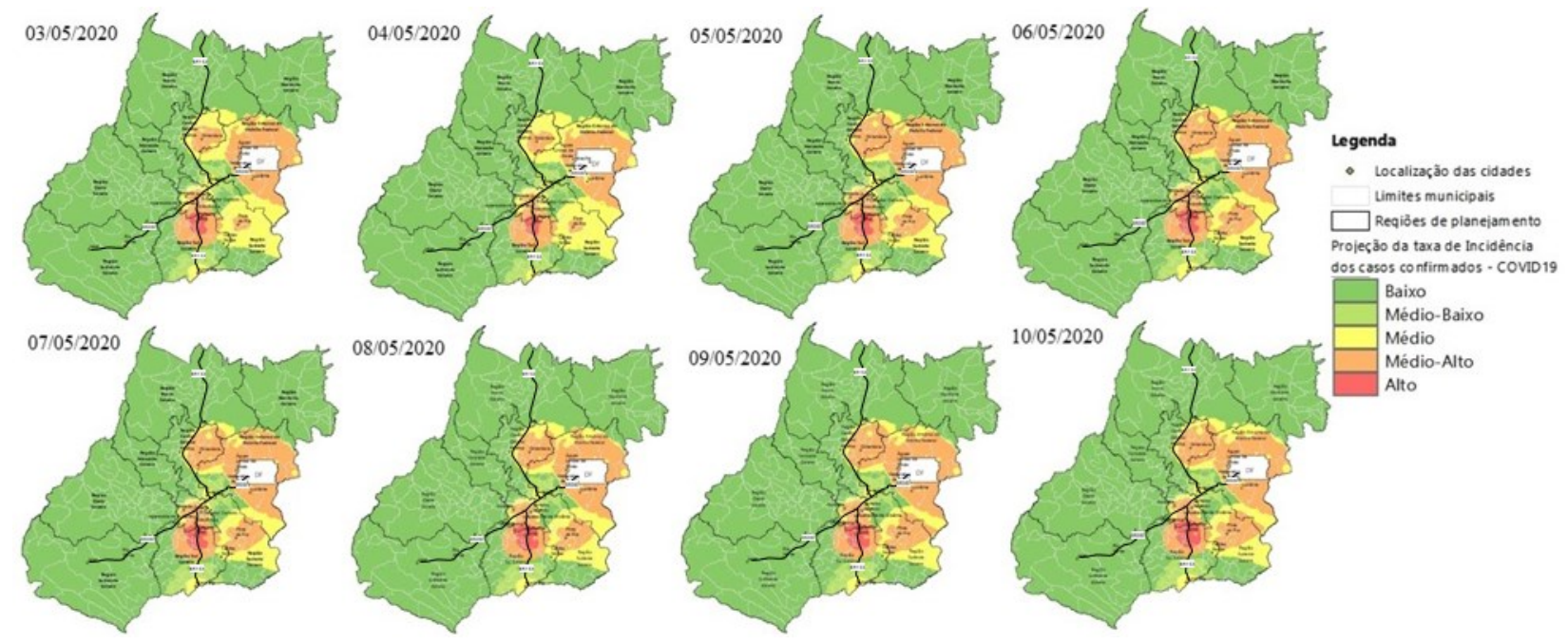

Os modelos preditivos demonstram-se bastante eficientes para dimensionar a progressão da doença, aspecto fundamental para ações de planejamento de medidas de saúde. Dentre os fatores importantes proporcionados com o entendimento em escala municipal, está a identificação das estruturas municipais de saúde, com alocação das ações considerando a disponibilidade principalmente de leitos hospitalares, leitos de UTI e respiradores (ou ventiladores) mecânicos. A identificação das infraestruturas médicohospitalares e suas respectivas capacidades, concomitantemente com a representação municipal da disseminação do vírus, favorece a ação direcionada com as especificidades municipais, norteando a manutenção, endurecimento ou flexibilização de ações de políticas públicas voltadas ao combate e prevenção do novo coronavírus.

Entre os componentes que afetam a compreensão deste estudo, está a ausência de um aprofundamento nas características demográficas dos municípios, como a identificação da população urbana e rural, a avaliação da densidade populacional, migrações internas e mobilidade urbana. A subnotificação dos casos interfere na precisão dos cálculos, pois os números adotados não necessariamente correspondem aos casos totais de pessoas portadoras do vírus, sobretudo em virtude dos indivíduos assintomáticos e da ausência de testes de confirmação para toda a população. Outros aspectos importantes são: reinfecção pela COVID-19 na mesma pessoa, embora evidências científicas atuais apontem que é uma probabilidade remota; o índice de adesão pela população aos decretos de combate e prevenção a novo coronavírus; infraestrutura urbana e o Índice de Desenvolvimento Humano (IDH).

\section{CONCLUSÃO}

Este trabalho possibilitou demonstrar espacialmente dados epidemiológicos da COVID-19 em Goiás de 18 de março a 01 de maio de 2020, identificar as regiões com maior intensidade da disseminação e fazer uma projeção da disseminação da doença por mais 10 dias. Os modelos preditivos demonstram-se bas- 
Análise espacial de risco e previsão de casos confirmados do

Bernard Silva de Oliveira Helber de Carvalho Covid-19 no estado de Goiás

tante eficientes para dimensionar a progressão da doença, aspecto fundamental para ações de planejamento de medidas de saúde. A compreensão espacial da propagação de epidemias possibilitada por uma leitura mais assertiva das características municipais, permiti nortear melhor as ações dos órgãos responsáveis.

\section{REFERÊNCIAS BIBLIOGRÁFICAS}

BDE-GOIÁS. Banco de Dados Estatísticos do Estado de Goiás. Sistema de Informações Estatísticas Relativas ao Estado de Goiás. Disponível em: https://www.imb.go.gov.br/. Acesso em: 10 abr. 2020.

CARDOSO, Phillipe Valente; SEABRA, Vinicius da Silva; BASTOS, Izabela Braz, COSTA, Evelyn de Castro Porto. A importância da análise espacial para tomada de decisão: um olhar sobre a pandemia de COVID-19. Revista Tamoios, [S.I.], v. 16, n. 1, maio 2020. ISSN 1980-4490. Disponível em: https://www.epublicacoes.uerj.br/index.php/tamoios/article/view/50440/33476. Acesso em: 11 nov. 2020. https://doi.org/10.12957/tamoios.2020.50440

CRODA, Julio Henrique Rosa; GARCIA, Leila Posenato. Resposta imediata da Vigilância em Saúde à epidemia da COVID-19. 2020. https://doi.org/10.5123/S1679-49742020000100021

FAROOQ, Muhammad; HAFEEZ, Abdul. Covid-resnet: A deep learning framework for screening of covid19 from radiographs. Disponível em: https://arxiv.org/abs/2003.14395. Acesso em: 13 mai. 2020.

GOIÁS (Estado). Decreto n ${ }^{\circ}$ 9.633, de 13 de março de 2020. Diário Oficial Estado de Goiás: suplemento, Goiânia, Goiás, ano 183, n. 23.257, p.1-2, 13 mar. 2020a.

GOIÁS (Estado). Decreto n ${ }^{\circ} 9.634$, de 13 de março de 2020. Diário Oficial Estado de Goiás: edição extra, Goiânia, Goiás, ano 183, n. 23.257, p.1-2, 14 mar. 2020 b.

GOIÁS (Estado). Decreto 9.637, de 17 de março de 2020. Diário Oficial Estado de Goiás: suplemento, Goiânia, Goiás, ano 183, n. 23.260, p. 1, 17 mar. 2020c.

GOIÁS (Estado). Secretaria de Estado da Saúde. Informe epidemiológico sobre o Coronavírus 2019 (COVID-19) - 27/03/2020. Goiânia: Secretaria de Estado da Saúde, 27 mar. 2020. Disponível em: https://www.saude.go.gov.br/files/banner_coronavirus/INFORMEEPIDEMIOLOGICOSOBREOCORONAVi RUS2019270320.pdf. Acesso em: 27 mar. 2020d.

IBGE - Instituto Brasileiro de Geografia e Estatística (IBGE). Estimativas da População. Rio de Janeiro. 2019. Disponível em: https://www.ibge.gov.br/estatisticas/sociais/populacao/9103-estimativas-depopulacao.html?edicao=25272\&t=downloads. Acesso em: 10 abr. 2020.

IMB - INSTITUTO MAURO BORGES DE ESTATÍSTICAS E ESTUDOS SOCIOECONÔMICOS. Malha municipal 2017. Goiânia: IMB, 2017. Disponível em:

http://dados.sieg.go.gov.br/Sieg/produtosIMB.asp?cod=4627 Acesso em: 10 abr. 2020.

IMB - INSTITUTO MAURO BORGES DE ESTATÍSTICAS E ESTUDOS SOCIOECONÔMICOS. GOIÁS visão geral - overview. Goiânia: IMB, 2018. Disponível em:

https://www.imb.go.gov.br/files/docs/publicacoes/goias-visao-geral/goias-visao-geral.pdf. Acesso em: 06 abr. 2020.

KOUBAA, Anis. Understanding the COVID19 Outbreak: A Comparative Data Analytics and Study. Disponível em: https://arxiv.org/abs/2003.14150. Acesso em: 13 mai. 2020.

OLIVEIRA, Ana Rita et al. Stochastic simulation model for the spatial characterization of lung cancer mortality risk and study of environmental factors. Mathematical Geosciences, v. 45, n. 4, p. 437-452, 2013. https://doi.org/10.1007/s11004-013-9443-8

ORGANIZAÇÃO PAN-AMERICANA DE SAÚDE - OPAS/OMS. Folha informativa - CovID-19 (doença causada pelo novo coronavírus). Disponível em:

https://www.paho.org/bra/index.php?option=com_content\&view=article\&id=6101:covid19\&ltemid=875. Acesso em: 22 abr. 2020. 

Helber de Carvalho

PENERLIEV, Milen; PETKOV, Veselin. Geodemographic Aspects of Covid-19. Espaço e Economia. Revista brasileira de geografia econômica, n. 18, 2020. https://doi.org/10.4000/espacoeconomia.13444

VARELLA, C. A. A.; JUNIOR, D. G. de S. Estudo do Interpolador IDW do Arcview para Utilização em Agricultura de Precisão. Universidade Federal Rural do Rio de Janeiro, Seropédica, Rio de Janeiro, 2008. Disponível em:

http://ufrrj.br/institutos/it/deng/varella/Downloads/IT190_principios_em_agricultura_de_precisao/Aulas/Est udo\%20do\%20interpolador\%20idw\%20arcview.htm. Acesso em: 18 abr. 2020. 